Supporting information for

\title{
Acoustically detonated microbubbles coupled with low frequency insonation: Multiparameter evaluation of low energy mechanical ablation \\ Mike Bismuth ${ }^{\dagger}$, Sharon Katz ${ }^{\dagger \ddagger}$, Hagar Rosenblatt ${ }^{\dagger}$, Maayan Twito ${ }^{\dagger}$, Ramona Aronovich ${ }^{\dagger}$, and Tali llovitsh ${ }^{\dagger *}$
}

† Department of Biomedical Engineering, Tel Aviv University, Tel Aviv 6997801, Israel

¥ The Sagol School of Neuroscience, Tel Aviv University, Tel Aviv 6997801, Israel

${ }^{*}$ Corresponding Author

E-mail address: ilovitsh@tauex.tau.ac.il 


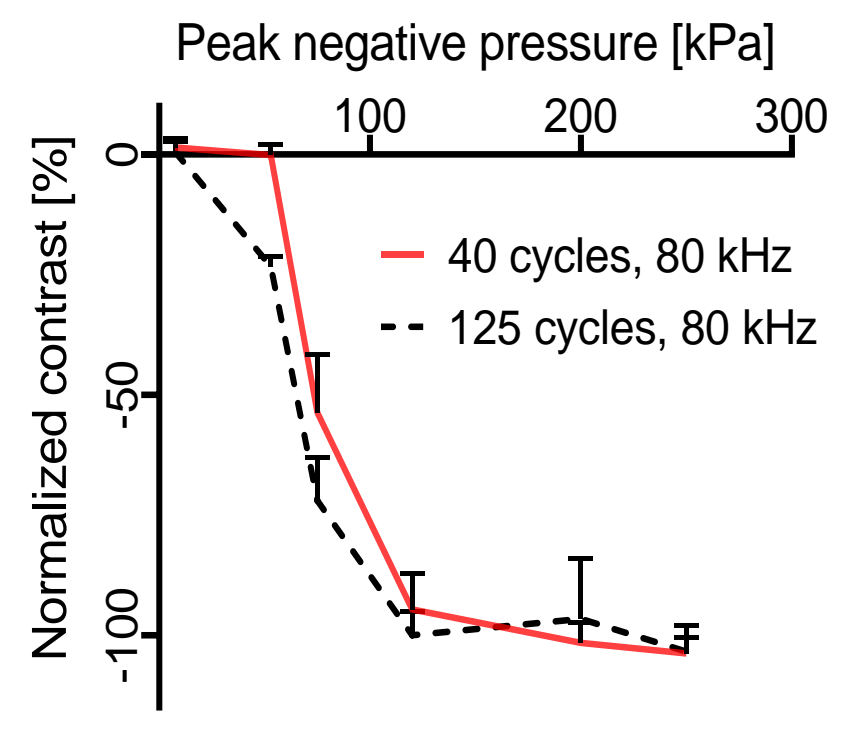

Figure S1. Normalized contrast reduction as a function of the peak negative pressure for $80 \mathrm{kHz}$ insonation with 40 cycles or 125 cycles, and a pulse repetition frequency of $30 \mathrm{~Hz}$. All experiments were performed in triplicates. All data are plotted as mean \pm SD. 


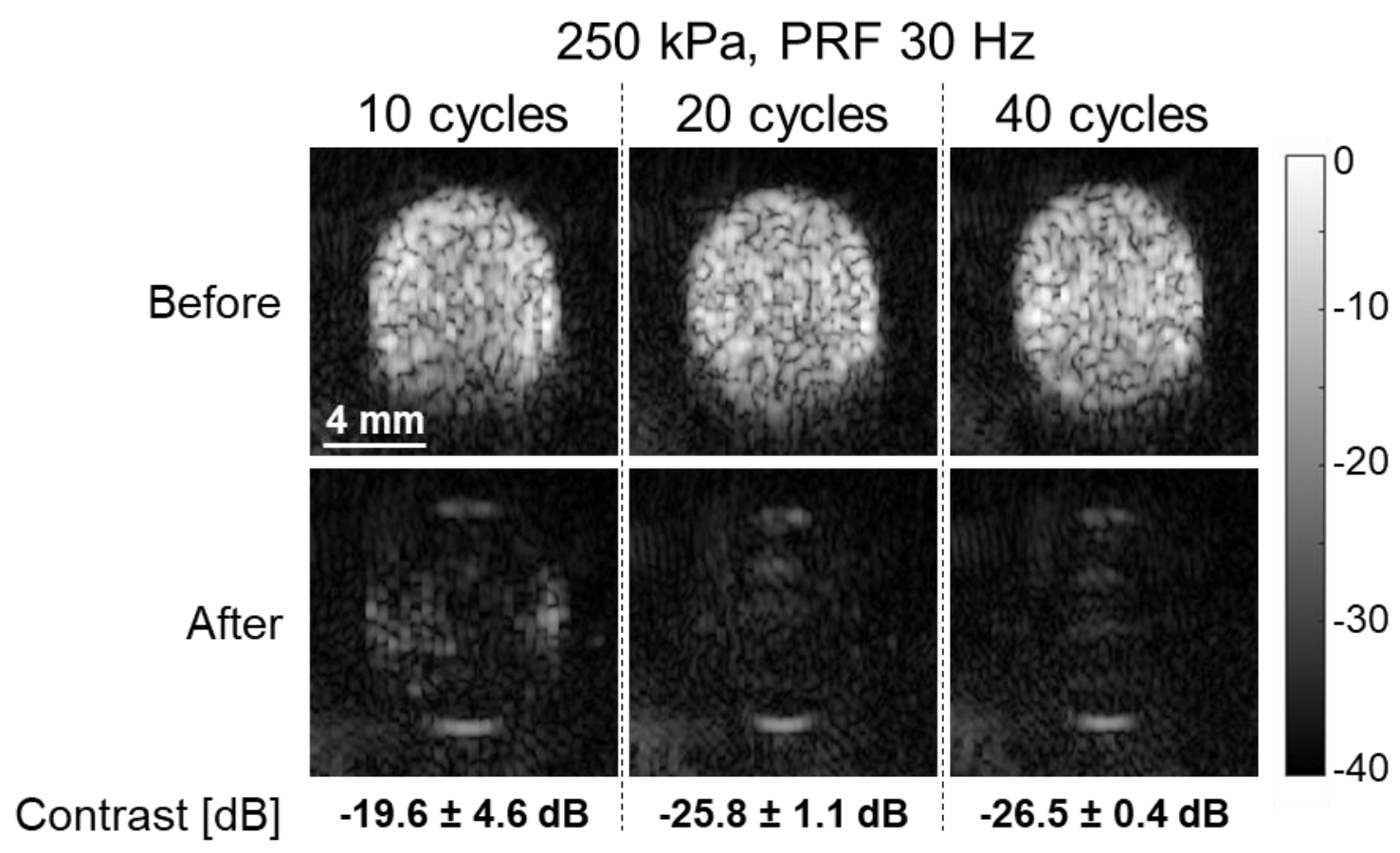

Figure S2. Optimization of the number of ultrasound (US) cycles in a tissue mimicking phantom. US images of a microbubbles-filled inclusion before and after application of a therapeutic US treatment with center frequency of $80 \mathrm{kHz}$, for number of cycles of 10,20 and 40 (duty cycles of $0.375 \%, 0.75 \%$ and $1.5 \%$, respectively). Constant parameters are peak negative pressure of $250 \mathrm{kPa}$ and pulse repetition frequency (PRF) of $30 \mathrm{~Hz}$. All experiments were performed in triplicates. All data are reported as mean $\pm \mathrm{SD}$. 


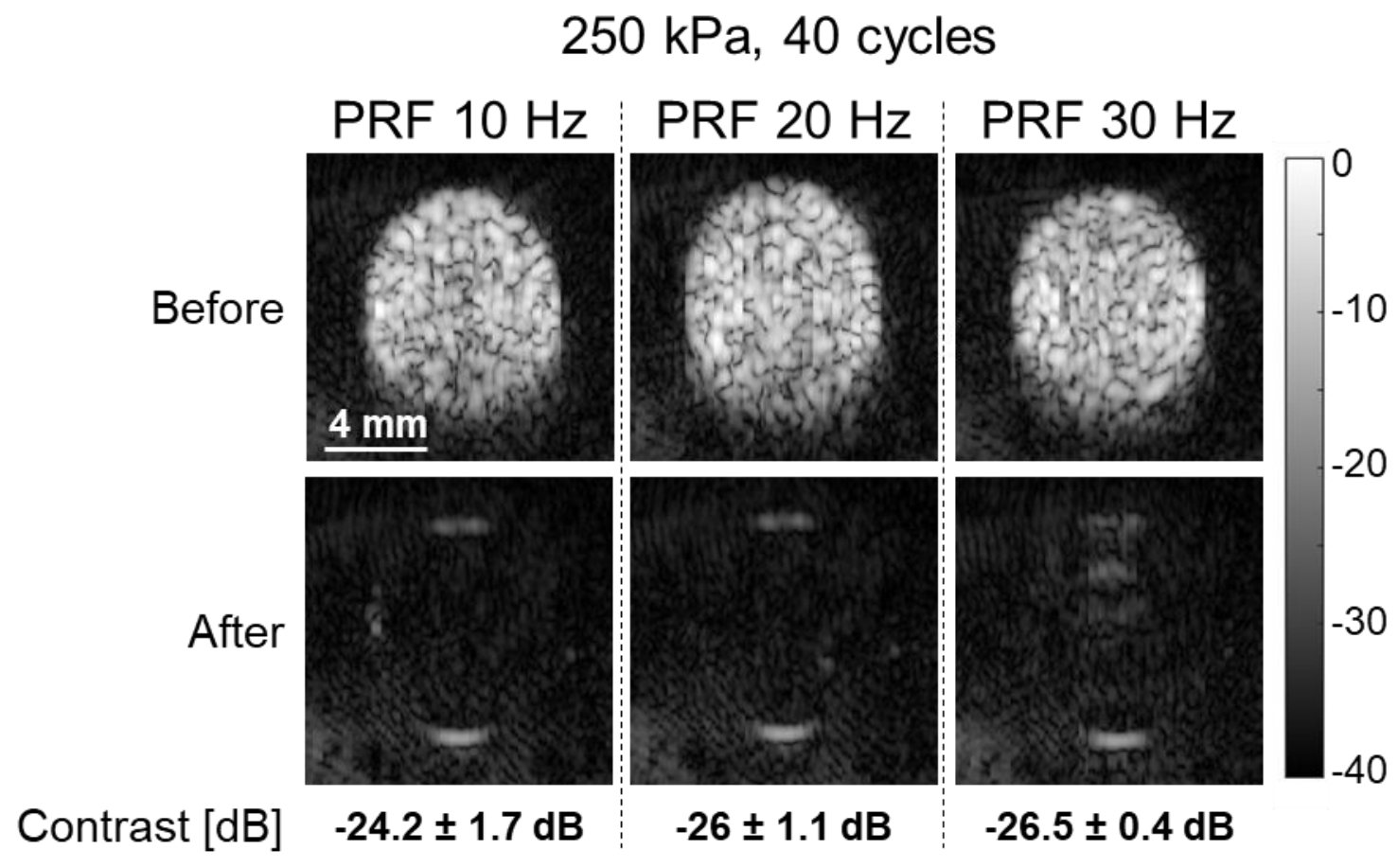

Figure S3. Optimization of the pulse repetition frequency (PRF) in a tissue mimicking phantom. Ultrasound (US) images of a microbubbles-filled inclusion before and after application of a therapeutic US treatment with center frequency of $80 \mathrm{kHz}$, for PRFs of 10,20 and 30 (duty cycles of $0.5 \%, 1 \%$ and $1.5 \%$, respectively). Constant parameters are peak negative pressure of $250 \mathrm{kPa}$ and pulse length of 40 cycles. All experiments were performed in triplicates. All data are reported as mean \pm SD. 